\title{
Proceedings of the second meeting of the British Neurosurgery Research Group, Chollerford, Northumberland, 20-21 April 1995
}

IMPAIRIDI PRESSURE AUTOREGUI ATION AND CO RLACTIVITY WITH EARLY HYPOPERFUSION AFTIER SEVERE INJURY IN A RODINT MODEL OF NLUROTRAUMA

IR Piper, D Weir, JD Miller. Clinical Neurosciences, Western General Hospital, Edinburgh, UK

Earlier work showed that cerebral blood flow (CBF) after severe injury using a rodent model of neurotrauma was raised by four hours after injury. This work has been extended to test whether pressure autoregulation and $\mathrm{CO}_{2}$ reactivity is affected at this time and also whether CBF is influenced at an earlier time point of 15 minutes after injury.

Anaesthetised Sprague-Dawley rats $(\mathrm{n}=$ 18) received either sham or a $450 \mathrm{~g} \times 2 \mathrm{~m}$ weight drop injury, regional $\mathrm{CBF}$ using $\left[{ }^{1+} \mathrm{C}\right]$ iodoantiprine quantitative autoradiography (QAR) $(\mathrm{n}=8)$ was used for assessing CBF at 15 minutes after injury, and the hydrogen clearance $(\mathrm{H} 2)$ technique $(n=10)$ for repeated half hourly measurements. After injury the $\mathrm{H} 2$ group were instrumented for ICP measurement. Blood pressure was raised or lowered using methoxamine infusion or phlebotomy respectively. To test $\mathrm{CO}_{2}$ reactivity arterial $\mathrm{PaCO}_{2}$ was increased to $80-90 \mathrm{~mm} \mathrm{Hg}$ through adding $5 \% \mathrm{CO}_{2}$ in the inspired gases.

All animals after injury became hypotensive $(40-60 \mathrm{~mm} \mathrm{Hg}$ ) returning to preinjury levels within 10 minutes. QAR data showed hypoperfusion at 15 minutes after injury in all 10 brain regions measured. Reduced $\mathrm{CBF}$ as a percentage of baseline ranged from $-18 \%$ in cerebellum to $-51 \%$ in occipital cortex. Pressure autoregulation studies in sham injured animals showed a significant correlation between cerebral perfusion pressure (CCP) and cerebrovascular resistance $(\mathrm{CVR}=\mathrm{CCP} / \mathrm{CBF})$ over a $\mathrm{CCP}$ range from $25 \mathrm{~mm} \mathrm{Hg}$ to $110 \mathrm{~mm} \mathrm{Hg}(r=$ $0.8222, \mathrm{P}<0.01)$. This relation was lost in the severe injury group. $\mathrm{CO}_{2}$ reactivity was also significantly depressed $(P<0.05)$ in the severe injury group $(0.551)$ compared with the sham injury group $(1.66 \mathrm{ml} / 100$ $\mathrm{g} / \mathrm{min} /$ torr)

1 Piper IR, Kelly PAT, Statham PFX, et al. $\mathcal{f}$ Neurotrauma 1993;10:562.

CEREBRAL HAEMODYNAMIC RESPONSE TO A SHORT TIERM COMPRISSSION OH THE COMMON CAROTII) ARTERY: BIG TLBE FLOW $\mathrm{I}$

COR'TICAL CIRCUIATION

HK Richards, M Czosnyka, JD Pickard. MRC Cambridge Centre for Brain Repair and Academic Neurosurgery Unit, Addenbrooks Hospital, Cambridge, UK

The components involved in the cerebrovascular responses invoked by carotid artery compression were assessed using an anaesthetised rabbit model.

In $12 \mathrm{New}$ Zealand white rabbits, basilar artery (BA) blood flow velocity (FV), cortical laser Doppler flux (LDF), and arterial blood pressure (AP) were recorded during 30 second compressions of the common carotid artery. $\mathrm{PaCO}_{2}$ was changed in steps from $25 \mathrm{~mm} \mathrm{Hg}$ to $65 \mathrm{~mm} \mathrm{Hg}$. Compressions at each $\mathrm{PaCO}_{2}$ level were repeated at least three times.

CBF was analysed during 89 compressions. LDF showed a significant $(P<0.001)$ decrease just after compression, then a compensatory increase during the duration of compression $(P<0.05)$. After release transient hyperaemic increase in LDF was observed $(P<0.0001)$. The relative magnitude of this increase was significantly correlated with $\mathrm{PaCO}_{2}(r=-0.38 ; \mathrm{P}<0.01)$. BA blood flow velocity exhibited a significant increase during compression with an equivalent immediate decrease after the release ( $P$ $<0.0001)$. The magnitude of these responses was not significantly correlated with Pa(c).

In conclusion, during compression of the carotid artery, a decrease in cortical microcirculation is seen. It is partially compensated by autoregulatory vasodilatation, which produces transient hyperaemia after release. The level of this vasodilatation depends on the baseline $\mathrm{PaCO}_{2}$. An acceleration in blood inflow along the BA during compression has a mainly passive character: its magnitude is not dependent on arterial $\mathrm{PaCO}_{2}$.

THE REI ATION BETWIIEN TRANSCRANIAL DOPPLER (TCD) PULSATII ITY AND CEREBROVASCULAR RESIS'IANCE Marek Czosnyka, Hugh Richards, Helen Whitehouse, John D Pickard. MRC Cambridge Centre for Brain Repair and Academic Neurosurgery Unit, Addenbrooks Hospital, Cambridge, UK

To clarify the relation between TCD pulsatility and cerebrovascular resistance (CVR) an increase in the $\mathrm{PaCO}_{2}$ was compared with a decrease in CPP autoregulated animals.

12 New Zealand white rabbits were studied under licence of the UK Animals (Scientific Procedures) Act, 1986. Rabbits were prepared and anaesthetised using methods previously described.' TCD in basilar artery, laser Doppler cortical perfusion, arterial pressure, end-tidal $\mathrm{CO}_{2}$, and ICP were measured continuously.

Four groups were analysed: where $\mathrm{PaCO}_{2}$ was altered (in the range $25-45 \mathrm{~mm} \mathrm{Hg}$ ), where gradual haemorrhagic hypotension was used to reduce CPP, where the ganglion blocking drug trimetaphan was injected to induce a transient hypotension, and where intracranial hypertension was produced by subarachnoid saline infusion.

During the hypercapnic challenge there was a positive correlation between CVR and PI $(r=0.77 ; \quad P<0.0001)$. In all three groups where CCP was reduced correlation between CVR and PI was negative $(r=$ $-0.84 ; \mathrm{P}<0.0001)$. While a rise in $\mathrm{CO}_{2}$ acts mainly as a vasodilator of cerebral arterioles, a decrease in CCP decreases the tension of vascular musculature of the whole arterial bed, decreasing CVR and increasing its compliance. PI is dependent cumulatively on CVR and compliance of cerebral arteries.

1 Czosnyka M, Richards H, Kirkpatrick P, Pickard JD. Neurosurgery 1994;35:287-93.

A NEW MODEI, OF ISCHAEMIA IN-VITRO USING ORGANOTYPIC HIPPOCAMPAL SIICE CLITURES AK Pringle, GJC Wilde, LE Sundstrom, F Iannotti. Clinical Neurosciences, Southampton University and Southampton General Hospital, Southampton, UK

To fully determine the mechanisms of neuronal death after ischaemia, many cell culture models have been developed. However, all of these fail to reproduce the characteristics of ischaemia in vivo. Using a new cell culture technique a model which better represents ischaemia in vitro has been characterised.

Hippocampal slices were prepared from 8-10 day old rats and maintained in culture on semiporous membranes for two weeks Hypoxia was induced by removing oxygen through saturation of culture medium with $95 \% \mathrm{~N}_{2} / 5 \% \mathrm{CO}$. After hypoxia cultures were returned to normoxic conditions for 24 hours. Ischaemia was simulated by combining hypoxia and glucose free medium. Cell death was determined by propidium iodide fluorescence which is only detectable in dead cells, and also by histological techniques.

Significant damage was detected in the CA region following 80 minutes of hypoxia. 60 minutes of simulated ischaemia produced a more severe lesion than hypoxia alone with damage detectable in both $\mathrm{CA}$ and $\mathrm{CA}_{3}$. Hypoxic damage was attenuated by the sodium channel blocker tetrodotoxin (TTX) and the NMDA receptor antagonist MK 801 if present during the insult, but only by TTX if added posthypoxia. Ischaemic dam age was prevented by TTX, MK-801, and CNQX (an AMPA receptor antagonist) if present during the insult, but only MK-80 added postischaemia.

It is concluded that this represents a valid in vitro model of transient global ischaemia which will permit further elucidation of biochemical aspects of ischaemic neurotoxicity. 
PROTECTIVE EFFECT OF TUMOUR NECROSIS FACTOR IN AN IN VITRO MODEL OF TRANSIENT GLOBAL ISCHAEMIA

GJC Wilde, AK Pringle, LE Sundstrom, F Iannotti. Clinical Neurosciences, Southampton University, Southampton General Hospital, Southampton, UK

Tumour necrosis factor (TNF) has been shown to increase after injury to the hippocampus, and to protect cultured embryonic rat hippocampal neurons against metabolic and excitotoxic insults. The effects of TNF were studied in an in vitro model of ischaemia using organotypic hippocampal slice cultures (OHSC) which maintain the organisational features of brain tissue in a defined environment.

To stimulate the effects of ischaemia, cultures were exposed to glucose free medium saturated with $95 \% \mathrm{~N}_{2} / 5 \% \mathrm{CO}_{2}$ for $60 \mathrm{~min}$ utes containing the fluorescent exclusion dye propidium iodide (PI). Cultures were then returned to normal conditions in the presence of PI. Cell damage was monitored by PI uptake 24 hours after insult, followed by histological processing to determine cell morphology. Cultures were preincubated for 24 hours with TNF and it was present throughout ischaemia and the recovery period. Cell death was expressed as the percentage of the area represented by the $\mathbf{C A}_{1}$, $\mathrm{CA}_{3 / 4}$, and dentate gyrus exhibiting fluorescence. Statistical significance was determined using Student's $t$ test.

After ischaemia $49 \cdot 1(46) \%(n=13)$ of the total area of cell layers exhibited PI fluorescence. A dose dependent neuroprotective effect was seen with TNF, with the maximum concentration of TNF tested (100 $\mathrm{ng} / \mathrm{ml}$ ) producing highly significant protection, reducing PI fluorescence to $9 \cdot 3(1 \cdot 2) \%$ of total area $(n=7, P<0.001)$.

TNF protects against ischaemic insult in vitro in a dose dependent manner. The $\mathrm{ED}_{50}$ of $0.9 \mathrm{ng} / \mathrm{ml}$ is comparable with that previously reported to protect from excitotoxic insults.

USE OF JUN PEPTIDE STAINING IN ASSESSING THE DEGREE OF INJURY AFTER OPTIC NERVE STRETCH

J Wasserberg, WL Maxwell, DI Graham. Departments of Neurosurgery and Neuropathology, Southern General Hospital, Glasgow, UK

The study investigated the use of JUN as a possible marker of neuronal injury in the guinea pig optic nerve stretch model of diffuse axonal injury.

JUN is a transcription factor thought to be involved in cellular repair mechanisms and is expressed in rat retinal ganglion cells (RGCs) after severe optic nerve injury.? Using immunocytochemical staining of retina it was possible to visualise retinal ganglion cells by JUN positive staining.

There is a baseline expression of JUN in the retinal ganglion cells of control guinea pigs which does not occur in control rats. Qualitative levels of JUN expression were increased over baseline up to three weeks after stretch injury. A much greater staining intensity was seen in surviving RGCs after optic nerve crush but statistically non-significant cell loss after stretch.

It is concluded that JUN expression is not a marker of injury but acts as a marker of surviving RGCs. The finding of sustained
JUN expression combined with insignificant cell loss provides indirect evidence of intrinsic neuronal recovery after stretch injury.

1 Gennarelli TA, Thibault LE, Tipperman R, et al. F Neurosurg 1989;71:224-53.

2 Koistinaho J, Hicks KJ, Sagar SM. $f$ Neurosci Res 1993;34:250-5.

DIABETES AND NITRIC OXIDE: EVIDENCE FOR A POTENTIAL ASSOCIATION IN THE REDUCTION OF BASAL CEREBRAL BLOOD FLOW IP Fouyas, PAT Kelly, IM Ritchie, R Whittle. Department of Clinical Neurosciences, Western General Hospital, Edinburgh, UK

The purpose of this investigation was to measure local cerebral blood flow (LCBF) in insulin dependent, spontaneously diabetic (BB) rats to assess firstly, whether the disease processes result in altered basal LCBF and secondly whether nitric oxide (NO) pathways might be involved in any dysfunction of cerebrovascular mechanisms.

Experiments were performed on diabetic $(\mathrm{DP}, \mathrm{n}=8)$ and diabetes resistant (DR, $\mathrm{n}=$ 8) $\mathrm{BB}$ rats. LCBF ( $\mathrm{ml} / 100 \mathrm{~g} / \mathrm{min}$ ) was measured using the fully quantitative $\left[{ }^{14} \mathrm{C}\right]$ iodoantipyrine autoradiographic technique ${ }^{1}$ 20 minutes after the intravenous injection of $N^{\mathrm{G}}$-nitro L-arginine methyl ester (L-NAME) (30 $\mathrm{mg} / \mathrm{kg}$ ) or saline. Data are presented as mean (SD).

No differences in plasma glucose, $\mathrm{PCO}_{2}$, $\mathrm{PO}_{2}$, or $\mathrm{pH}$ were found between groups, but base excess of $3.23(1.8)$ in DP rats confirmed the metabolic disturbances of diabetes. In saline injected DP rats LCBF was significantly lower in most regions compared with DR controls (for example, hippocampus $72(8) v 92$ (8); globus pallidus $54(6) v$ 73 (2)). Injections of L-NAME reduced LCBF in most brain areas in DR rats (for example, hippocampus 59 (4)), but had no significant effect in the DP group (for example, hippocampus $67(10)$ ).

This study shows that LCBF is reduced in diabetes. However, the effects are regionally heterogeneous, possibly reflecting differential susceptibility to the disease in a subpopulation of cerebral blood vessels. The attenuated response to L-NAME in diabetic rats is consistent with an involvement of the NO pathway in the observed perturbation of basal LCBF.

1 Sakurada O, Kennedy C, Jehle J, et al. Am $\mathcal{F}$ Physiol 1978;234:H487-99.

NERVE GROWTH FACTOR IN HUMAN CEREBRAL BLOOD VESSELS

L Chadwick, A Parrett, P Anand, DV Sinicropi, RE Williams-Chestnut, PJ Hamlyn. Department of Neurosurgery and Department of Neurology, The Royal Hospitals NHS Trust, London, UK, and Department of Medicinal and Analytical Chemistry, Genentech Inc, San Francisco, USA

Nerve growth factor (NGF) concentrations in human blood vessels and its distribution around the circle of Willis were measured for the first time to determine its possible role in the innervation of these vessels. The subjects were seven cadavers with no known neurological disorder (mean age 72 years, mean postmortem delay 23 hours). The concentration of NGF was measured by a specific enzyme linked immunoassay. Concentrations were as follows: anterior cerebral $0.82 \mathrm{ng} / \mathrm{g}$; internal carotid 0.78 $\mathrm{ng} / \mathrm{g}$; posterior cerebral $2.02 \mathrm{ng} / \mathrm{g}$; superior cerebellar $2.14 \mathrm{mg} / \mathrm{g}$, and posterior communicating artery $1.87 \mathrm{mg} / \mathrm{g}$. The concentrations of NGF were thus significantly lower in the anterior compared with the posterior cerebral vessels in the circle of Willis $(\mathbf{P}<$ $0.04-0.015)$. There was also a significant age related decline.

In conclusion, NGF concentrations were lower in the anterior than in the posterior circle, this was inversely related to the concentrations of peptides as identified by some members of this group (NPY, VIP, SP, and CGRP). The concentration also decreased with age. The relation of the peptides NPY, $\mathrm{SP}$, and CGRP to NGF is being investigated by further studies.

CHARACTERISATION OF TRANSIENT FOCAL DEPOLARISATIONS IN EXPERIMENTAI STROKE WITH IN VIVO NADH FLUORESCENCE IMAGING Spencer P Harland, Anthony J Strong, Brian S Meldrum, Diana J Withington. Department of Neurology and Neurosurgery, The Institute of Psychiatry and Maudsley Hospital, London, UK

Transient focal depolarisations (TFDs) resembling spreading depression in boundary zones in focal ischaemia promote maturation to irreversible infarction. Using processed fluorescence images, we have located the onset and tracked the spread of transient cortical phenomena with the characteristics of TFDs after middle cerebral artery occlusion (MCAO). Four cats were anaesthetised with chloralose. The right MCA territory was exposed and then occluded transorbitally. The cortex was illuminated at $370 \mathrm{~nm}$; cortical fluorescence was imaged with an intensified CCD camera and the sequences of images digitised. In each sequence the first image was subtracted from all subsequent ones in the sequence, and pseudocolour-coded difference images prepared.

The method yields information about the origin, propagation, and frequencies of TFDs, but their frequency and amplitude vary between experiments. The available data presented suggest a transient reduction of $\mathrm{NAD}^{+}$in the $\mathrm{SG}$ and posterior marginal gyrus (MG), but oxidation when a TFD reaches the anterior MG. Propagation rates are close to the range for spreading depression. Pre and post-TFD blood flow data suggest little difference in flow either side of the transient zone.

MANAGEMENT OF CEREBRAL PERFUSION PRESSURE IN SEVERELY BRAIN INJURED PATIENTS

$\mathrm{KH}$ Chan, SCP Ng, JD Miller. Division of Neurosurgery, Department of Surgery, The University of Hong Kong, Hong Kong

The optimal cerebral perfusion pressure (CPP) for brain injury may vary with time and the patient. This study aims to define the role of induced increases in blood pressure (BP) in defining this CPP threshold by 
examining the responses of jugular venous oxygen saturation $\left(\mathrm{SJO}_{2}\right)$, middle cerebral artery (MCA) blood flow velocity and intracranial pressure (ICP).

Twenty three severely brain injured patients had continuous monitoring of BP, ICP, CPP, end-tidal carbon dioxide $\left(\mathrm{CO}_{2}\right)$ concentration, arterial oxygen saturation, $\mathrm{SJO}_{2}$, and MCA Doppler pulsatility index (PI). Data were collected every six seconds by a computer. BP was increased by phenylephrine $(20 \mathrm{mg}$ in $20 \mathrm{ml}$ saline) to raise the mean BP $45.5(27 \cdot 7) \mathrm{mm} \mathrm{Hg}$ (mean $(\mathrm{SD})$ ) above baseline. $\mathrm{CO}_{2}$ reactivity was examined after each test. Sixty one increased BP tests were performed (median of three tests/patient).

Analysis of the relation between $\mathrm{SJo} / \mathrm{CPP}$ and PI/CPP, showed a breakpoint at CPP of $70 \mathrm{~mm} \mathrm{Hg}$. Below CPP of $70 \mathrm{~mm} \mathrm{Hg}$, significant correlation existed between SJo, $(r$ $=0.61, \mathrm{P}<0.001)$, PI $(r=0.53, \mathrm{P}<0.001)$ and CPP. Above CPP of $70 \mathrm{~mm} \mathrm{Hg}$, no correlation existed. The lower the baseline CPP level, the greater the increase in $\mathrm{SJO}_{2}(\mathrm{P}$ $<0.05)$ and decrease of PI $(P<0.001)$. Three patterns of response were noted: (1) Baseline CPP and ICP at $55.6(11.8)$ and $26.4(6.9) \mathrm{mm} \mathrm{Hg}$ respectively $(\mathrm{n}=32)$, increased BP resulted in decrease of ICP $(-4.2(5.6) \mathrm{mm} \mathrm{Hg}, \mathrm{P}<0.001)$, increase in $\mathrm{SJO}_{2}(6.7(5.3) \%, \mathrm{P}<0.001)$ and decrease in PI $(-0.25(0.20), \mathrm{P}<0.001)$; (2) Baseline CPP and ICP at $79.9(8.8)$ and $21.4(6.4)$ $\mathrm{mm} \mathrm{Hg}$ respectively $(\mathrm{n}=22)$, there was a significant decrease in ICP $(-2.6(4.8) \mathrm{mm}$ $\mathrm{Hg}, \mathrm{P}<0.05)$, but no changes in SJo, $(2.0$ $(5 \cdot 4) \%$, NS) and PI $(-0.04(0 \cdot 15)$, NS) suggesting intact autoregulation: (3) Baseline CPP and ICP at $41.9(16.6)$ and $50.7(24.5)$ $\mathrm{mm} \mathrm{Hg}$ respectively $(\mathrm{n}=7)$, increased BP resulted in a parallel increase of ICP $(23.7$ $(14.6) \mathrm{mm} \mathrm{Hg}, \quad \mathrm{P}<0.001)$ so that CPP $(10.0(26.7) \mathrm{mm} \mathrm{Hg}, \quad \mathrm{NS}), \quad \mathrm{SJO}) \quad(-5.4$ $(13.8) \%$, NS) and PI $(-0.56(1.17)$, NS remained unaltered. The last pattern was only observed in patients who lost $\mathrm{CO}_{2}$ reactivity.

In conclusion, an induced increase in BP can be used to define the optimal CPP and as part of ICP reduction therapy after severe brain injury.

APPLICATIONS OI: PROTON MAGNITIC RISSONANCE SPIECIROSCOPY AT THE ROYAI PRESTON HOSPITAI

AP Jones, WJ Gunawardena, CMA Coutinho, CHG Davis. North Western Medical Physics Department, Department of Clinical Radiology, and Department of Neurosurgery, Royal Preston Hospital, Preston, UK

Localised proton magnetic resonance spectroscopy is a new form of MR spectroscopy that can be used as an investigative procedure on patients. Previous experience with other forms of MR spectroscopy has led to premature unsubstantiated conclusions on cerebral metabolites, which are being challenged. Localised $1 \mathrm{H}$ MRS permits the noninvasive in vivo analysis of the structure. Relative concentration in chemical surroundings of biologically important molecules in a given tissue within the brain spectra can be obtained for specific volumes of tissue down to a size as small as $5 \times 5 \times$ $5 \mathrm{~mm}$. Furthermore, the new accurate localisation techniques used in MRS allow the specificity of an examination to be increased if standard MR imaging is correlated with the spectroscopic information.

The initial difficulties in collecting data were described. The main course of the work has been in the study of motor neuron disease. However, the data have also been collected from a patient with a giant MS plaque and two patients with tumours. Intensive investigations revealed metabolic changes in creatinine/phosphocreatinine $(\mathrm{Cr} / \mathrm{Pcr})$, choline $(\mathrm{Cho}), \mathrm{N}$-acetyle aspartate (NAA), and lactate relative to normal brain and these results correlated well with the theoretical effects of the pathology. Decreases in NAA associated with neuronal loss and increases in the lactate resulting from glycolysis have been observed and preliminary work suggests that this form of spectroscopy may become the gold standard for metabolic investigation of cerebral disease.

CAN NIAR INFRAREI) SPI:CTROSCOPY DFETECI CHAVGES IN REGIONAL OXYHALMOGLOBIN DUE TO ILNCTIONAL ACTIVATION?

TJ Germon, CW Pleydell-Pearce, P Evans, N Barnett, P Watt, RJ Nelson. Department of Neurosurgery, Frenchay Hospital, Burden Neurological Institute, Bristol and Johnson and Johnson Medical, UK

Specific verbal tasks increase left frontal cortical cerebral blood flow.' The increase is in excess of the metabolic requirement for oxygen, leading to an increase in local oxyhaemoglobin $(\mathrm{OHb})$. This specific response has been studied using multichannel near infrared spectroscopy (NIRS) to determine the sensitivity of NIRS to these intracranial changes.

Seven volunteers performed a previously validated, ${ }^{5}$ verbal activation task while relative changes in $\mathrm{OHb}$ concentration were monitored using novel, eight channel NIRS. The changes in $\mathrm{OHb}$ were analysed with respect to side, time course, reproducibility, and distance of receiving optode from the NIR source.

(1) There was no significant difference between the right and the left sides in any channel. (2) The mean maximum increase in $\mathrm{OHb}$ occurred between 60 and $100 \mathrm{sec}-$ onds after the start of the activation task. (3) The change was not reproducible by a second performance of the tasks in reverse order. (4) The increase in $\mathrm{OHb}$ between tasks reduced as optode separation increased.

The bilateral increases in $\mathrm{OHb}$ in all eight channels (when PET shows a strictly left sided response); the reduction in the size of the change with increasing optode separation (which is characteristic of extra rather than intracranial NIR light attenuation), the lack of reproducible results and the time course of the response (slower than would be predicted from transcranial Doppler activation studies) all suggest that the major influence on the NIRS signal was extracranial. This work confirms previous findings that extracranial NIR light attenuation has a major influence on the detection of changes in intracranial oxygenation. ' These effects must be overcome if NIRS is to be used to study functional activation.

Frith $\mathrm{CD}$, Friston K, Liddle PF, Frackowiak RSJ. Proc $R$ Soc Lond B 1991;244:241-6. Germon TJ, Kane NM, Manara AR, Nelson RJ. Br F Anaesth 1994;73:503-6.
IN"RAOPERATITIE (ORTICAL SOMATOSINNSORY IEVOKED POTENITAI MONITORING IS A USIIILI. TOOL IN COMPIIAX CERVICAL SLRGERY

Daniel May, H Alan Crockard, Stephen J Jones. The National Hospital, Queen Square, London, UK

Intraoperative monitoring of somatosensory evoked potentials (SEP) is a well established method in scoliosis surgery to prevent neurological damage. The purpose of this study was to analyse the benefit of SEP monitoring in cervical surgery.

Bilateral median nerve stimulation was used and SEPs were recorded from the neck and scalp surface (cortical) in 186 patients undergoing surgery for cervical conditions as different as degenerative myelopathy, radiculopathy, instability, bone tumours, and extradural and intradural tumours. Seventy seven patients were in group 3 according to Ranawat's grading system. The clinical, electrical, and surgical findings were analysed comparing those patients with SEP alterations with those without. Parametric and non-parametric statistical analyses were performed.

In 32 instances there was a significant change in preoperative SEP defined as a sudden drop in amplitude of $50 \%$ or more which could not be accounted for by anaesthetic or other systemic factors. Eight patients had postoperative evidence of neurological worsening. Four others had SEP drops of fully reversible non-surgical origin. There were no "false negative" results. SEP alterations correlated positively with the level of surgery (high cervical $v$ mid and low cervical), the amount of bony work (vertebrectomy) and the presence of a preoperative neurological deficit. On the other hand no SEP modifications were encountered in simple anterior decompression surgery (Smith-Robinson).

In conclusion, despite the rate of "false positive", SEP monitoring enhanced the probability of detectirig neurological damage in cervical surgery. This is especially true in high risk situations where corrective forces are applied to the spine, where neurological deficits are present preoperatively, and in cord tumours.

COGNTIVE ASSLSSMIENI OI: PATIENTS BLIFORL: ANi) AFTER (AR('TII) IENI)ARTERECTOMY A PRELIMINARY RI:P(OR'I

Joanna L Iddon, Peter J Kirkpatrick, Barbara J Sahakian. University Department of Neurosurgery and Psychiatry, Cambridge University and Addenbrooke's Hospital, Cambridge, UK

"High resolution" neuropsychological tests can detect neurological impairment in injured brains before any structural changes are seen with conventional imaging methods. The aim of this study was to establish whether perioperative cerebral microemboli and/or haemodynamic changes cause cognitive deficits after carotid endarterectomy (CE).

Seven patients with symptomatic high grade stenosis who were neurologically intact were assessed for cognitive changes before and after CE. All patients completed the national adult reading test and verbal fluency as well as computerised tests of attention, visual and spatial working memory, and planning taken from the Cambridge 
neuropsychological test automated battery (CANTAB). Preoperative and postoperative assessments were carried out within one week of surgery.

Initial analysis of accuracy data indicated that there were no differences in six of the nine neuropsychological parameters measured preoperatively when compared with age and IQ match controls. In addition postoperative scores showed no significant change to the preoperative assessments for the group.

These preliminary results indicate that uncomplicated CE does not cause any significant neuropsychological deficits. 1 Sahakian BJ, Owen AM. $\mathcal{F}$ R Soc Med 1992;85:
399-402.

PREOPERATIVE CARBON DIOXIDE REACTIVITY DOES NOT PREDICT THE CEREBRAL

HAEMODYNAMIC CHANGES DURING CAROTID ENDARTERECTOMY

PJ Kirkpatrick, P Smielewski, S Nair, M Czosnyka. University Department of Neurosurgery, Cambridge University, Cambridge, UK and Addenbrooke's Hospital, Cambridge, UK

The aim was to determine whether preoperative cerebrovascular reactivity studies give predictive information on the degree of cerebral haemodynamic compromise seen during carotid endarterectomy (CE).

Thirty three patients awaiting CE underwent preoperative cerebral carbon dioxide $\left(\mathrm{CO}_{2}\right)$ reactivity testing with transcranial Doppler (TCD). During surgery cerebral haemodynamic changes were monitored with TCD and near infrared spectroscopy (NIRS). Middle cerebral artery flow velocity (FV) and concentrations of oxy- $\left(\mathrm{HbO}_{2}\right)$ and deoxy-(Hb) haemoglobin were recorded, and total haemoglobin concentration $(\mathbf{t H b})$ calculated. Variables were normalised to baseline values, and mean values obtained for one minute epochs at 5,10 , and $15 \mathrm{~min}$ utes after internal carotid (IC) clamping The maximal signal changes at five minutes post clamping $\left(\mathrm{Max}^{5}\right)$, and the subsequen rate of recovery $\left(\mathrm{I}^{\mathrm{rec}}\right)$ towards baseline were calculated for $\mathrm{FV}, \mathrm{HbO}_{2}, \mathrm{Hb}$, and $\mathrm{tHb}$

The $\mathrm{Max}^{5}$ for $\mathrm{HbO}_{2}$ and $\mathrm{Hb}$ were inversely related $(r=0.471, P=0.016)$. There was no relation between the preoperative $\mathrm{CO}_{2}$ reactivity value and the indices of $\mathrm{Max}^{5}$ and $\mathrm{I}^{\mathrm{rec}}$ for any variable measured.

Preoperative cerebral $\mathrm{CO}_{2}$ reactivity does not accurately predict the intraoperative haemodynamic compromise produced during $C E$, and provides no indication of the collateral reserve responsible for recovery of haemodynamics after IC cross clamping.

CEREBROVASCULAR REACTIVITY IN PATIENTS WITH CAROTID STENOTIC DISEASE: STUDY USING TCD AND NEAR INFRARED SPECTROSCOPY

P Smielewski, M Czosnyka, P Kirkpatrick, H Whitehouse, JD Pickard. MRC Centre for Brain Repair and Academic Neurosurgical Unit, Cambridge, UK

$\mathrm{CO}_{2}$ reactivity in healthy volunteers ${ }^{1}$ showed high correlation between TCD and near infrared spectroscopy parameters (NIRS, Hamamatsu 1000). The aim was to study the TCD and NIRS reactivities and relate them to the percentage of carotid artery stenosis in a group of 93 patients. The examination protocol consisted of five minutes baseline recording followed by six minutes increased $\mathrm{EtCO}_{2}$. The cerebrovascula reactivity indices were calculated as follows: TCD -percentage change in FV per $1 \mathrm{kPa}$ change in $\mathrm{EtCO}_{2}$; NIRS -absolute change in concentration of oxygenated $\left(\mathrm{HbO}_{2}\right)$, and deoxygenated haemoglobin $(\mathrm{Hb}[\mu \mathrm{mol} / 1])$ per $1 \mathrm{kPa}$ change in $\mathrm{EtCO}_{2}$

The average cerebrovascular reactivities were: TCD: $14 \cdot 7 \% / \mathrm{kPa}$ (SD 7.9); $\mathrm{HbO}_{2}$ $1.65 \mu \mathrm{mol} / \mathrm{l} / \mathrm{kPa}$ (SD 0.83); $\mathrm{Hb}:-1.14$ $\mu \mathrm{mol} / 1 / \mathrm{kPa}(\mathrm{SD} 1 \cdot 1)$. Correlation analysis of the results showed: (1) a significant correlation between reactivity in TCD and NIRS ( $=0.51, P<0.001$ ); however, the correlation is weaker than in normal subjects. ${ }^{1}(2)$ Reactivity in TCD and NIRS was significantly correlated with the degree of stenosis in the ipsilateral ICA but there was no correlation with the stenosis in the ECA.

1 Smielewski P, Minhas P, Zayat S El, Kirkpatrick P, Czosnyka M, Pickard JD Cerebrovasc Dis 1994;4(suppl 3):23.

SPECT EVALUATION OF HUMAN BRAIN TUMOURS USING THALLIUM AND TYROSINE MARKERS

G Cruickshank, A Mitchener, J Patterson, K McKellar, J Owens, DM Hadley. Department of Neurosurgery and Clinica Physics, Institute of Neurological Sciences, Glasgow, UK

Malignant brain tumours consist of foca malignant tumour and diffuse tumour with a high malignant potential, responsible for the high (greater than $80 \%$ ) local recurrence.

Efforts to treat focal disease by surgery and focused radiotherapy can be effective at prolonging survival, but newer treatments must consider the diffuse component as well.

Using dual labelling of tumours with thallium and tyrosine SPECT which indicate only viable tumour cells, it has been possible to visualise the two tumour components. Such image directed approaches to viable, rather than morphological tumour, (as displayed by CT and MRI), provide not only a basis for monitoring responses of malignant tumours, but also the novel possibility of determining the extent of diffuse tumour and the possibility of assessing the response of low grade tumour to therapy.

DO EXPERIMENTAL GLIOMA DECREASE SEIZURE THRESHOLDS?

A Beaumont, $M$ Clarke, IR Whittle. Department of Clinical Neurosciences, Western General Hospital, Edinburgh, UK

Generalised or partial seizures are a common problem with many supratentoria gliomas. Their underlying pathophysiological mechanisms are poorly understood. To investigate this problem clinical and EEG seizure thresholds were investigated in experimental rodent gliomas using the epileptogenic drug pentylenetetrazole (PTZ).

Mixed C6: A15A5 gliomas were grown in adult Wistar rats after unilateral stereotactic implantation of a 50:50 cell mix. One control group had intracerebral injection of tissue culture medium $(10 \mu \mathrm{l})$ only. Animals were studied between 14 and 16 days later under $\alpha$-chloralose and urethrane anaesthesia. Four channels of EEG (raw and spectrally analysed) were recorded from brain surface using platinum ball electrodes placed in the frontal and parietal regions. Baseline EEG (15 minutes) and continuous EEG during and after (30 minutes) PTZ (Sigma) infusion $(100 \mu \mathrm{l} / \mathrm{min})$ were recorded. The nature of and time to appearance of seizure manifestations after starting PTZ were also noted.

Fourteen animals were studied (five normal, five with tumours, four sham implants). Mean BP, $\mathrm{PaCO}_{2}, \mathrm{PaO}_{2}$ and temperature were similar in the three groups. All tumours were in the posterior caudatoputamen/hippocampal region and were large. Baseline EEG showed predominately slow wave activity irrespective of electrode placement. After PTZ infusion a sequence of vibrissal twitching (following a mean of $14.5 \mathrm{mg} / \mathrm{kg}$ PTZ); fore/hind limb twitches $(17.5 \mathrm{mg} / \mathrm{kg})$, myoclonic jerking $(46 \mathrm{mg} / \mathrm{kg})$; nasal twitches $(47 \mathrm{mg} / \mathrm{kg})$; and status $(77 \mathrm{mg} / \mathrm{kg})$ was observed in all animals. The seizure thresholds for all phenomena except status were highest and significantly different in the tumour bearing animals. Baseline control processed EEG showed predominance of delta and increased alpha/beta with PTZ. Tumour animals showed more fluctuation and greater power in baseline processed EEG and variable responses after PTZ infusion (increased delta in $45 \%$, decreased delta $25 \%$ ). These studies have confirmed that experimental gliomas alter baseline EEG and the EEG and behavioural response to PTZ. Rather surprisingly the tumour raised the threshold for many seizure manifestations.

HYPOXIA AND MALIGNANT BRAIN TUMOURS: SIMULTANEOUS DYNAMIC

MICROPOLAROGRAPHY AND MICRO LASER DOPPLER RECORDING OF BLOOD FLOW AT OPERATION

GS Cruickshank, K Seex, R Duckworth. Department of Neurosurgery and Neuroanaesthesia, Institute of Neurological Sciences, Glasgow, UK

Malignant brain tumours are resistant to radiotherapy and part of their resistance may be due to the high levels of hypoxia in these tumours recorded in vivo using fine needle dynamic micropolarography.

In an attempt to characterise the relevance of factors influencing these recordings, dynamic micropolarography recordings have been combined with multichannel micro laser Doppler recording of tumour and peritumoural microcirculatory blood flow at operation.

The effects of altering blood pressure and arterial carbon dioxide were presented. These results showed an intrinsic temporal fluctuation in microregional tumour blood flow, that could result in temporal and spatial variations in microenvironmental oxygenation. The variation of $\mathrm{PO}_{2}$ profiles with impressed fluctuations in $\mathrm{PaCO}_{2}$ or $\mathrm{BP}$ resulted in changes which confirm that intracranial tumour hypoxia is of a significant level to diminish the effects of radiotherapy and chemotherapy. 
DISTRIBUTION AND FUNCTION OF NITRIC OXIDE MECHANISMS IN EXPERIMENTAL GLIOMA

IR Whittle, FM Collins, IM Ritchie, PAT Kelly. Department of Neurosciences, Western General Hospital, Edinburgh, UK

Nitric oxide (NO) is important as both a signalling molecule in vascular smooth muscle and as a "killer" molecule used by activated immune cells. This preliminary study was undertaken in experimental rat $\mathrm{C} 6$ gliomas to evaluate the distribution of $\mathrm{NO}$ synthase and to assess vasoregulative functions of NO within the tumour.

Gliomas were grown for 10-14 days in adult Wistar rats after intracerebral implantation of cultured $\mathrm{C} 6$ cells. On the day of the experiments, equal numbers of $\mathrm{C} 6$ recipients were injected iv with either the nitric oxide synthase inhibitor $N^{\mathrm{G}}$-nitro-L-arginine methyl ester (L-NAME) (30 mg/kg; $\mathrm{n}=7$ ) or saline $(n=7)$, and local cerebral blood flow (LCBF) was measured using the fully quantitative $\left[{ }^{14} \mathrm{C}\right]$-iodantipyrine autoradiographic technique. NO synthase activity (NOS) was assessed in adjacent sections by NADPH-diaphorase histochemistry.

All 14 tumours were NOS positive, and although the distribution within each tumour was heterogeneous, levels were higher than in surrounding brain. In LCBF studies $\mathrm{pH}, \mathrm{PCO}_{2}, \mathrm{Po}_{2}$, temperature, and plasma glucose were similar in saline and LNAME groups; arterial blood pressure was significantly higher and heart rate significantly lower in L-NAME treated rats. LCBF was significantly lower in the L-NAME group compared with controls in four out of the six areas measured contralateral to the tumour (average reduction 20\%). Within the tumours there was a highly significant reduction (average $40 \%$ ) in blood flow after administration of L-NAME.

This study identifies NOS activity in experimental glioma, but the contribution from infiltrating macrophages, tumour cells, and vascular endothelium is not known. Whatever the source of the NO, tumour blood flow seems to be more susceptible than normal brain to the effects of L-NAME.

GENE THERAPY FOR GLIOMA; CONCEPTUALLY SIMPLE, PRACTICALLY DIFFICULT?

L Mingwei, WL Kimber, IR Whittle. Department of Clinical Neurosciences, Western General Hospital, Edinburgh, UK

The initial description of gene therapy for the experimental 9L rodent gliosarcoma, using herpes viral thymidine kinase gene transfection and gancyclovir therapy was rapidly followed by clinical application. ${ }^{1}$ There are, however, many practical and technical problems in extrapolating experimental studies using a single cell line to studies of human malignant glioma. Differences in transfection rates of some relevant genes into a range of rat and human glioma cell lines, using different techniques, were performed in vitro to evaluate potential difficulties in gene therapy for glioma.

The five glioma cell lines C6, A15A5 (rodent); Go-G-CCM, A172, CCF-STTG1 (human), and the 3T3 fibroblast were evaluated for transfection efficiency using three different liposomes (DOTAP; lipofectin; lipofectamine) and the Lac $Z$ gene (CMV beta-galactosidase). The influence of cell density and DNA-liposome ratio was evaluated. Variations in clonal sensitivity to gancyclovir after stable transfection with ptgCMVHyg/ thymidine kinase were also studied and clones with high expression used to quantify the "bystander" effect. The ease of transfection was found to vary widely between different cell lines. Transfection was most efficient using lipofectamine but was related to cell proliferation rate and also accompanied by significant cell toxicity. Further results concerning the other studies were presented.

These preliminary in vitro studies suggest that even using optimal in vitro conditions there are major differences in transfection rates in glioma cell lines. These findings suggest there may be major practical difficulties in using gene therapy for gliomas.

Culver KW, Ram Z, Wallbridge S, et al. Science 1992;256:1550-2.

JUST HOW SILENT IS THE RIGHT HEMISPHERE? A-M Thomson, $\mathbf{R}$ Taylor, D Fraser, IR Whittle. Department of Clinical Neurosciences, University of Edinburgh, UK

In recent years the right cerebral hemisphere has been demonstrated to play a "minor" part in communication. Whereas the dominant hemisphere controls comprehension and expression of language the non-dominant hemisphere contributes to the understanding of idioms and humour and non-verbal aspects of communication such as facial expression and intonation.

When the only available test of right hemisphere language-the "right hemisphere language battery"-was used to assess 20 patients with right hemisphere brain tumours and 20 normal controls there was no statistically significant difference between the two sets of scores. Twenty seven patients with right and 53 patients with left hemisphere tumours were then assessed using the "western aphasia battery" which is a test of left hemisphere damage. The mean scores obtained before neurosurgery in the writing subtest were identical in both left and right tumour groups-20, maximum score 34 . The postsurgery mean scores were also identical; both showed an improvement of 6 points. The word fluency subtest produced a preoperation mean score of 15 in the right hemisphere tumour group, 6 points below the norm. These results unexpectedly showed that expressive language impairments can occur with a right hemisphere lesion. Neurosurgeons should be aware that right hemispheric brain surgery can affect language function. Problems with current assessment tools will be discussed.

MANAGEMENT AND OPERATIVE OUTCOME FROM SUBARACHNOID HAEMORRHAGE IN TWO REGIONAL CENTRES

AD Mendelow, T Hope, I Chambers, V Orpe, S Wilson, J Stevenson, RP Sengupta, PJ Crawford, A Jenkins, NV Todd, J Firth, J Punt. Newcastle General Hospital, Newcastle, UK and Queen's Medical Centre, Nottingham, UK

The comparison between management and operative outcome on discharge in Newcastle and Nottingham has been reported previously. ${ }^{1}$ These results indicated a higher percentage of favourable outcomes (good recovery and moderate disability) on discharge from Nottingham than from
Newcastle for both overall management and for surgery. However, these differences on discharge were affected by bed availability and length of stay.

The six month outcomes are now reported for a series of patients admitted in $1990 / 1 / 2$. A total of 524 were admitted to Newcastle and 317 to Nottingham. Favourable outcomes occurred in $77 \%$ in Newcastle and $84 \%$ in Nottingham. However, for grade I and II (WFNS) patients, favourable outcomes were $89 \%$ in Newcastle and $86 \%$ in Nottingham, reflecting a lower good grade rebleed rate (GGRR) in Newcastle than in Nottingham $(4.8 \% v$ $7 \cdot 5 \%$ ). In Newcastle 337 patients underwent surgical treatment ( $86 \%$ favourably) compared with 198 in Nottingham (94\% favourably). For grade I and II patients the favourable six month postoperative outcomes were $91 \%$ and $95 \%$ respectively. In general, surgery was earlier in Newcastle than in Nottingham.

Assessment of outcome at six months rather than at discharge indicates fewer real differences between the two units.

1 Stevenson JC, Mendelow AD, Hope T, et al. Proc Am Assoc Neurosurg 1993.

VARIATIONS IN THE SYSTEMIC PATTERN OF CYTOKINE PRODUCTION AFTER SUBARACHNOID HAEMORRHAGE

RH Lye, WR Lamb, PEC Brenchley. University of Manchester and Department of Neurosurgery, Manchester Royal Infirmary, Manchester, UK

Cytokines are released after aneurysmal subarachnoid haemorrhage (SAH).

Immunoassay of daily blood samples from SAH patients enabled measurement of cytokines TNFa, IL-6, and IL-1 $\beta$. Concentrations of the IL- 1 receptor antagonist and soluble receptors for IL-6 and TNF (p55 and p75) were also measured Cytokine response of whole blood to endotoxin stimulation measured each patient's reserved ability to produce cytokines.

Eight patients underwent clipping of an aneurysm. None displayed systemic release of cytokines but endotoxin stimulation in vitro showed alterations in cytokine response during the postoperative period. Cytokine production increased (two patients) decreased (three patients), or oscillated (three patients) after surgery.

There was some correlation with clinical condition and outcome. Time series analysis suggested suppression of the oscillatory pattern around the time of operation which corresponded to improvement of clinical grade All patients exhibited less ability to secret TNFa after operation $(P<0.05)$ with an increase in soluble TNF receptors (p55 and p75) but little alteration of the IL-1 a or IL-6 preoperative response.

These preliminary findings disclose an alteration in the production of TNFa and its soluble receptors after SAH.

TWENTY FOUR HOUR SAMPLING OF NEUROPEPTIDE LEVELS IN HUMAN CS

L Chadwick, P Milner, G Burnstock, PJ Hamlyn. Department of Neurosurgery, St Bartholomew's Hospital and Department of Anatomy and Developmental Biology, University College, London, UK

Many groups assay vasoactive substances in CSF samples to investigate the cause of neu- 
rological diseases, but their results have been inconsistent. The fluctuation in neuropeptides has been investigated in human CSF over a period of 24 hours in controls and the disease state subarachnoid haemorrhage to assess the use of single time CSF specimens.

CSF samples were taken every hour for 24 hours from a lumbar drain. There were four control patients (three men, one woman) mean age 56.5 (14) years having had transphenoidal surgery or a dural repair and four subarachnoid haemorrhage patients (three women, one man) mean age 45.8 $(8 \cdot 6)$ years. Levels of endothelin, vasoactive intestinal polypeptide, neuropeptide $\mathrm{Y}$, calcitonin gene-related peptide, and substance $P$ were measured by enzyme linked immunoabsobent assay and checked with high performance liquid chromatography.

There was great variability in the levels of neuropeptides examined throughout the day in patients from both groups with no obvious pattern, with the exception of neuropeptide $\mathrm{Y}$ where there was a trend for higher levels in those patients with delayed ischaemic deficit.

Whereas the level of neuropeptide $\mathrm{Y}$ in CSF may be higher in patients with delayed ischaemic deficit, the results of any single time CSF experiment should be interpreted with caution.

GENE TRANSFER TO CEREBRAL VESSELS DURING VASOSPASM USING RECOMBINANT ADENOVIRUS

MG Muhonen, DG Martin, DD Heistad, MJ Welsh. Division of Neurosurgery, University of Iowa, Iowa City, USA

Vasospasm remains a major cause of morbidity and mortality after aneurysmal subarachnoid haemorrhage. Preliminary feasibility studies of gene therapy were explored using a viral vector to prevent spasm of cerebral vessels by transducing a reporter gene into cerebral vessels. Vasospasm was induced in mongrel dogs ( $\mathrm{n}$ $=6$ ) using the double haemorrhage intracisternal injection model. Basilar artery diameter was assessed by angiography and profound vasospasm was confirmed at four and seven days. Recombinant adenovirus $\left(1 \cdot 2-3 \cdot 8 \times 10^{10}\right.$ infectious units) expression nuclear-targeted $\operatorname{Iac} Z$ (reporter gene) under the control of the cytomegalovirus promoter was injected intracisternally at the same time as the induction of vasospasm in eight separate animals. The brains were removed and histochemically examined by staining for nuclear $\beta$-galactosidase. At two to seven days, $\beta$-galactosidase was expressed in leptomeninges over the brain stem, cortex, cerebral arteries, in small vessels in the cerebellum and brain stem, and in the ependymal lining of the ventricles. Gene transduction occurred within the adventitia of blood vessels, but none was observed in the smooth muscle or endolithium of the vessels.

The findings indicate that intracisternal injection of the recombinant adenovirus can be used for gene transfer to cerebral vessels and overlying meninges, even in the presence of cisternal blood and vasospasm. Transfer and expression of genes that encode for enzymes with vasodilator function (especially highly diffusible substances such as nitric oxide synthase) to cerebral blood vessels using recombinant viral vec- tors may be useful for prevention or treatment of cerebral vasospasm.

CHANGES AND SIGNIFICANCE OF

CALMODULIN, THROMBOXANE A2, AND MALON-DIALDEHYDE IN CSF AND SERUM OF PATIENTS WITH SEVERE HEAD INJURY Fei Zhou, Yi Sheng Yu, J Douglas Miller. Department of Clinical Neurosciences, University of Edinburgh, Scotland, UK

Calcium ions and prostaglandin metabolic changes in head injury have been the subject of some investigation in recent years. ${ }^{12}$ In this study, the CSF levels of calmodulin (CaM) and thromboxane B2 (TXB2) and serum levels of malon-dialdehyde (MDA) and superoxide dismutase (SOD) were observed in 60 patients with severe head injury in the first 7 days after injury to investigate the relation of the imbalance of calcium ions and the alterations of $\mathrm{CaM}$, thromboxane A2 (TXA2), MDA, and SOD levels with the occurrence of secondary brain damage. The therapeutic effects of nimodipine and cerebrolysin on this relation were also studied. $\mathrm{CaM}$ level was raised in the first seven days postinjury $(P<0.01)$ and reached a peak value within the first 24 hours. TXB2 and MDA levels were higher than normal in the first seven days $(P<$ 0.01 ) and reached a peak on the third day postinjury. SOD activity was below normal in the first seven days $(P<0.01)$. Patients with brain contusion exhibited a higher level of CaM, TXB2, and MDA than those with extradural haematoma. Treatment with cerebrolysin restored $\mathrm{CaM}$ to the normal level and decreased the TXB2 and MDA below normal on the seventh day postinjury whereas administration of nimodipine resulted in the normal level of $\mathrm{CaM}$ on the seventh day and that of TXB2 and MDA on the third day postinjury. Besides, the clinical outcome of the patients as assessed by GOS treated with cerebrolysin and nimodipine was better than those treated conventionally ( $\mathrm{P}<0.01)$.

These findings suggest that the imbalance of calcium ions in the neurons after head injury is an important factor for the occurrence of secondary brain damage and nimodipine and cerebrolysin have great potential for the treatment of head injury.

1 Takayasu M, Shibuya M, Kanamori M, et al. $\mathcal{F}$ Neurosurg 1985;63:417-9.

2 Shohami E, Shapira Y, Cotev S, et al. 7 Cereb Blood Flow Metab 1988;22:859-63.

USE OF MAGNETIC RESONANCE ANGIOGRAPHY IN THE ASSESSMENT OF EC-IC ANASTOMOSIS SS Praharaj, AD Mendelow, A Coulthard, A Gholkar. Departments of Surgery (Neurosurgery), Newcastle General Hospital and Radiology, RVI and Associated Hospitals Trust, Newcastle-upon-Tyne, UK

Six patients underwent EC-IC bypass procedures for the following conditions in which the internal carotid artery (ICA) required occlusion: two for intracavernous ICA giant aneurysms, three with base of skull tumours encompassing the ICA, and one with an ICA occlusion in whom the acetazolamide stimulation test had confirmed a poor collateral reserve in the ipsilateral hemisphere Each patient had a postoperative MRA study. Three of these patients also had conventional angiography for comparison.
There was a good correlation between conventional and MR angiography. MRA has the added advantage of allowing determination of direction of blood flow and by placing selective presaturation bands across the circle of Willis, the contribution of the EC-IC bypass solely to the cerebral circulation can be determined.

In conclusion, MR angiography is a safe and non-invasive method of evaluating patients who have had an EC-IC bypass procedure.

SUDDEN AGONISING HEADACHE. WILI

INCREASED AWARENESS OF ITS SIGNIFICANCE AMONG DOCTORS AFFECT THE OUTCOME OF SUBARACHNOID HAEMORRHAGE?

Christos M Tolias, Munchi S Choksey. Department of Neurosurgery, Walsgrave Hospital NHS Trust, Coventry, UK

Coventry and Warwickshire is a well defined area with around 750000 people. No significant change has occurred in this population over the past 10 years.

Using the angiography records of Walsgrave Hospital and the clinical audit data and postmortem reports from all four hospitals in our region all cases of subarachnoid haemorrhage (SAH) were identified from 1986 to 1995 . There were 610 patients: 310 were admitted after referral to the neurosurgical unit, 170 remained under the care of the physicians, and 130 died suddenly in the community.

The neurosurgical outcome of SAH by clinical grade on admission was consistent with other reported series. ${ }^{1}$ Of all patients with SAH identified in this region more than $34 \%$ reported "sudden agonising headache" in the weeks to days before major bleed. Only $18 \%$ of these were eventually referred to neurosurgery, although more than two thirds had sought medical advice. As a result only $72 \%$ of them were in grade I and II on admission. By contrast, $95 \%$ of patients who did not have any symptoms of a warning leak were in grades I and II.

A case controlled, prospective study involving the Coventry and Warwickshire region will be conducted over a five year period. General practitioners and casualty officers will be asked to refer all patients with sudden agonising headache to hospital. All referrals will be monitored and the validity, outcome, and cost benefit of the exercise will be assessed.

1 Haley EC, Kassell NF, Alves W, et al. $\mathcal{f}$ Neurosurg 1995;82:786-90.

CALCITONIN GENE RELATED PEPTIDE: WHERE NEXT?

BA Bell, JP Holland. Atkinson Morley Neurosciences Centre, St George's Hospital Medical School, London, UK

Calcitonin gene related peptide (CGRP) is widely distributed in the body. In the larynx CGRP and substance $P$ fibre distribution mirrors mast cells, and CGRP is involved in laryngeal oedema and the airway hyperaemia that occurs in asthma. In the heart the positive inotropic and chronotropic effects of CGRP resemble $\beta$-adrenoceptor agonists such as isoprenaline. In the stomach CGRP containing nerves occur around mucosal arterioles which connect with the T8-11 dorsal root ganglia, and CGRP dilates resistance supply arteries. This effect is ablated 
by nitric oxide (NO) blockers and CGRP inhibits gastrin and pepsin secretion, acid secretion, and gastric motility. CGRP is part of the stomach's neural emergency system, which requires an intact coeliac ganglion, and is triggered by mucosal barrier disruption (for example, ingestion of alcohol) leading to acid penetration of the mucosa, CGRP, and NO release (NO simulates mucous secretion), submucosal vasodilation, and enhancement of mucosal resistance. In the skin CGRP deficiency underlies Raynaud's phenomenon and is involved in the erythema of sunburn. In the bladder it modulates the micturition reflex via its sensory arm and urinary retention results if CGRP is depleted. In synovial joints CGRP increases blood flow and increases oedema in inflamed joints, and is increased in the afferent nerves of joints affected by mycobacterial arthritis. CGRP antibodies lessen the severity of experimental arthritis.

The ophthalmic trigeminal ganglion contains numerous CGRP-immunoreactive cell bodies and the nerve fibres distribute around ipsilateral branches of the circle of Willis to form the trigimenocerebrovascular system. The headache of a histamine infusion requires an intact trigeminal nerve, and trigeminal stimulation induced facial flushing is blocked by CGRP antagonists. Jugular venous CGRP increases in migraine, and sumatriptan (serotonin $S_{1}$ receptor agonist) restores the levels to normal. Jugular venous CGRP is increased after SAH in consort with the degree of vasospasm, and patients dying within 10 days of subarachnoid haemorrhage (SAH) show depleted CGRP levels in the middle cerebral artery (MCA). CGRP seems to be part of the mechanism to restore constricted cerebral vessel diameter that operates in migraine and $\mathrm{SAH}$, and in a pilot study of $13 \mathrm{SAH}$ patients given an IV infusion of $<1.15 \mu \mathrm{g} / \mathrm{min}$ CGRP, facia flushing occurred in nine, seven improved their WFNS grade with CGRP, and none improved with placebo. In a subsequent study, 62 patients received a CGRP infusion of $0.6 \mu \mathrm{g} / \mathrm{min}$ and 41 stopped treatment, 19 because of adverse events, 17 because they did not improve by four hours, four by lack of improvement beyond four hours, and one at the request of the patient. Only a third of the patients completed treatment but the relative risk of poor outcome in the 62 who received CGRP fell to 0.88 (95\% CI 0.60 to 1.28) when compared with 55 control patients.

In a pilot study of CGRP levels in CSF after SAH in 10 patients, seven with a good outcome had a mean level of $19.6 \mathrm{pg} / \mathrm{ml}$ and three with a poor outcome level of 6.9 $\mathrm{pg} / \mathrm{ml}$. The four patients with vasospasm had a mean level of $11.7 \mathrm{pg} / \mathrm{ml}$, and the six with no vasospasm had a level of $21.9 \mathrm{pg} / \mathrm{ml}$ In three patients in a coma after SAH given a $20 \mu \mathrm{g}$ intrathecal bolus of CGRP, their mean Glasgow coma score rose from 6 to 9 . In a rat MCA ischaemia model a $200 \mathrm{ng} / \mathrm{kg}$ intrathecal bolus of CGRP doubled CBF in the ischaemic area for over one hour and reduced the volume of ischaemic injury by $57 \%$.

A multicentre trial of intrathecal administration at the time of aneurysm surgery to replenish deficient CGRP stores on the adventitial surface of cerebral vessels may now be indicated. 